\title{
Acceleration and evolution of a hollow electron beam in wakefields driven by a Laguerre-Gaussian laser pulse
}

\author{
Guo-Bo Zhang, ${ }^{1,2}$ Min Chen,,${ }^{1, ~ *}$ C. B. Schroeder, ${ }^{3}$ Ji Luo, ${ }^{1}$ Ming Zeng, ${ }^{1}$ Fei-Yu Li, ${ }^{4}$ Lu-Le \\ $\mathrm{Yu},{ }^{1}$ Su-Ming Weng, ${ }^{1}$ Yan-Yun Ma, ${ }^{2,}{ }^{+}$Tong-Pu Yu, ${ }^{2}$ Zheng-Ming Sheng, ${ }^{1,4}$ and E. Esarey ${ }^{3}$ \\ ${ }^{1}$ Key Laboratory for Laser Plasmas (MOE) and Department of Physics and Astronomy, \\ Shanghai Jiao Tong University, Shanghai 200240, China \\ ${ }^{2}$ College of Science, National University of Defense Technology, Changsha 410073, China \\ ${ }^{3}$ Lawrence Berkeley National Laboratory, Berkeley, California 94720, USA \\ ${ }^{4}$ SUPA, Department of Physics, University of Strathclyde, Glasgow G4 ONG, UK
}

(Dated: February 2, 2016)

\begin{abstract}
We show that a ring-shaped hollow electron beam can be injected and accelerated by using a Laguerre-Gaussian laser pulse and ionization-induced injection in a laser wakefield accelerator. The acceleration and evolution of such a hollow, relativistic electron beam are investigated through threedimensional particle-in-cell simulations. We find that both the ring size and the beam thickness oscillate during the acceleration. The beam azimuthal shape is angularly dependent and evolves during the acceleration. The beam ellipticity changes resulting from the electron angular momenta obtained from the drive laser pulse and the focusing forces from the wakefield. The dependence of beam ring radius on the laser-plasma parameters (e.g., laser intensity, focal size, and plasma density) is studied. Such a hollow electron beam may have potential applications for accelerating and collimating of positively charged particles.
\end{abstract}

PACS numbers: $52.38 . \mathrm{Kd}, 52.65 . \mathrm{Rr}, 52.35 . \mathrm{Mw}$

\section{INTRODUCTION}

Laser wakefield acceleration (LWFA) studies have made rapid progress in the last decades due to the tremendous acceleration gradient and the advancement of ultrashort, ultraintense laser technology, e.g., chirpedpulse application (CPA) [1-3]. The wakefield acceleration gradient normally scales with the plasma density as $E(\mathrm{~V} / \mathrm{m}) \approx 96 \quad n_{0}\left(\mathrm{~cm}^{-3}\right)$, which makes $\mathrm{GeV}$ electron beam acceleration within a fewcentimeters distance $[4,5]$. At present, much research is focused on the improvement of the beam qualities such as energy spread and transverse emittance. These are critical for applications of the LWFA electron beams. Important progress has been achieved both theoretically and experimentally. For example, detailed injection control is possible using colliding laser pulse[6], yielding low energy spread beams[7]. By using ionization injection in an LWFA, both beam energy spread and emittance can be improved[8-11]. In addition to beam quality, beam shaping, both in longitudinal or transverse directions, is important for some applications. Recently, there has been interest in hollow electron beam acceleration, which can be used for the collective acceleration of ions and positrons[12], collimation for proton acceleration[13], and hollow x-ray generation. Conventionally, a hollow electron beam is created with an inductive voltage adder or linear transformer

\footnotetext{
*Authors to whom correspondence should be addressed. Electronic mail: minchen@sjtu.edu.cn

${ }^{\prime}$ Authors to whom correspondence should be addressed. Electronic mail:yanyunma@126.com
}

device[14, 15]. However, due to the extremely small spatial scales of the laser-driven wakefield and the accelerated beam, the tunability of such an electron beam inside a wake is much more challenging, compared to conventional radio-frequency (RF) accelerator. Tailoring the wakefield structure is a possible way to generate and accelerate electron beams with special spatial structures. Combined laser modes have been recently suggested to generate tailored wake structures, enabling high quality electron acceleration and positron acceleration by tuning the transverse wakefields[16, 17]. Recently, single-mode intense Laguerre-Gaussian (LG) laser pulses have also been proposed as a driver both for particle acceleration and harmonic generation in laser-plasma interactions [1821].

As is well known that, the LG laser has a hollow transverse intensity profile and it carry well-defined orbital angular momentum (OAM). In previous studies, Vieira et al. have studied the linear and nonlinear wakefield$\mathrm{s}$ excited by LG lasers through simulations and a ringshaped wakefield was observed $[18,22]$. Positron acceleration by such wakefield has been demonstrated in multidimensional particle-in-cell (PIC) simulations.

In this paper, we concentrate our studies on a hol- low electron beam injection through the ionization injection method $[23,24]$ and beam acceleration by such ring-shaped wakefield driven by a LG laser. Especially, we focus on the formation and evolution of the hollow electron beam in the wakefield. Angular momentum evolution of electron beam in laser-plasma accelerators has been studied through simulations and experiments.[25] Although the angular momentum cannot directly transfer between LG laser and the electrons, we find that, in the process of electron ionization and subsequent trap- 

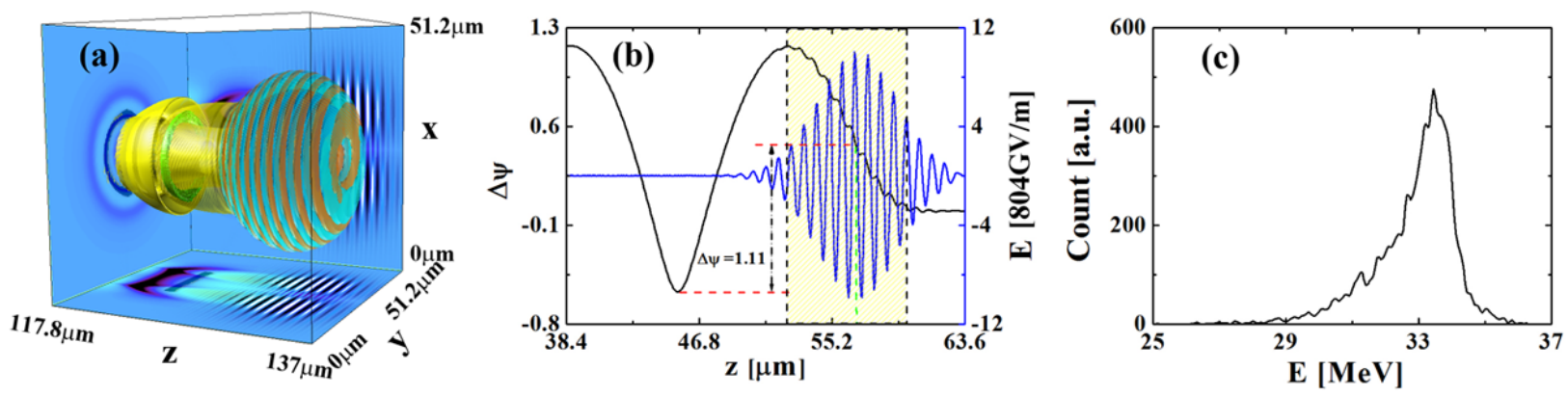

FIG. 1: (color online) (a) Spatial distribution of the hollow electron beam accelerated in the ring-shaped wake structure driven by an intense LG pulse. Green and yellow colors represent the charge density of the hollow electron ring and wake structure, respectively. The orange-blue colors represent the LG laser electric field. (b) Distribution of the laser electric field and the wake potential at $x=32.8 \mu \mathrm{m}$ and $y=25.6 \mu \mathrm{m}$. The yellow shaded region marks the area where the initial Nitrogen gas is located. (c) Energy spectrum of the electrons within the ring structure at $t=872 \mathrm{fs}$.

ping, the hollow electron beam can get angular momentum and rotate in the ring-shaped wakefield. Nevertheless, electrons at different positions of the hollow beam have different rotation characteristics due to two effects: the initial residual ionization momentum and the wakefield focusing force. As a result, the beam ellipticity evolves. The dependence of beam ring radius and charge on laser intensity, focal size and plasma density are studied by scaling simulations. Our studies describe a method to generate electron beams with special structures, e.g., hollow center, using a laser wakefield accelerator that can be tested on existing laser systems.

\section{GENERATION AND EVOLUTION OF A HOLLOW ELECTRON BEAM}

To study the acceleration and evolution of the hollow relativistic electron beam in detail, three dimensional particle-in-cell (3D-PIC) simulations have been performed. In our simulations, the normalized laser vector potential $\left(\boldsymbol{a}=e \mathbf{A} / m c^{2}\right)$ of the LG laser pulse with wavelength of $800 \mathrm{~nm}$ and mode of $(1,0)$ is given by:

$$
a(r, \xi)=a_{0} c_{1,0}\left(r / w_{0}\right) \exp \left(-r^{2} / w_{0}^{2}-\xi^{2} /(c \tau)^{2}+i \varphi\right) L_{0}^{1}
$$

Where $w_{0}=10.2 \mu \mathrm{m}$ and $\tau=17 \mathrm{fs}$ are the laser spot size and pulse duration, respectively. $L_{0}{ }^{1}=1$ is a Laguerre polynomial with radial index $p=0$ and azimuthal index $l=1, c_{1,0}=1$ is normalizing factor. We adopt the co-moving frame variables, $\xi=z-c t$ is the forward co-moving coordinate with the laser, $r=\sqrt{x^{2}+y^{2}}$ is the radial distance to the axis, $x$ and $y$ are the transverse coordinates, and $\varphi$ is the azimuthal angle. The dimensionless amplitude of the incident laser pulse is $a_{0}=2$, which corresponds to the laser peak intensity of $I=8.53 \times 10^{18} \mathrm{~W} / \mathrm{cm}^{2}$. The laser is linearly polarized along the $x$ direction and propagates along the $z$ direction. The simulation utilizes a moving window and includes an
ADK tunneling ionization module[26]. The initial neutral gas mixture is composed of $99 \%$ Hydrogen and $1 \%$ Nitrogen. The electron density of the initial hydrogen plasma is $n_{0}=6.96 \times 10^{18} \mathrm{~cm}^{-3}$. In order to get a clear picture of the dynamics of the ionization-injected hollow electron beam, the Nitrogen gas is only occupied within a short region (about $8 \mu \mathrm{m}$ ) for ionization injection[24]. The simulation box size is $51.2 \mu \mathrm{m} \times 51.2 \mu \mathrm{m} \times 25.6 \mu \mathrm{m}$ and has been divided into $400 \times 400 \times 400$ cells with $2 \times 2 \times 2$ macro-particles per cell.

A typical snapshot of produced hollow electron beam in the $3 \mathrm{D}$ simulations is shown in Fig. 1(a). As one can see, an annular electron structure (as indicated by the green color ring) is accelerated at the back of the first wakefield bucket (marked by the yellow color). The orange-blue colors show the electric field of the driver LG pulse. These electrons are ionized from the Nitrogen gas. Figure 1(b) shows the laser electric field and the wake potential at $x=32.8 \mu \mathrm{m}$ and $y=25.6 \mu \mathrm{m}$ corresponding to the peak wakefield position. The ionized electrons satisfy the ionization injection condition of $\Delta \psi>1[10,24,27]$, where $\psi$ is the wake potential and $\Delta \psi$ is the difference in wake potential between the ionization position and the back of the wakefield bucket. The particle tracer in the simulation also confirms the source of these injected electrons. The energy spectrum of the electrons within the ring structure is presented in Fig. 1(c), which is taken at $t=872 \mathrm{fs}$ before the electrons reach the dephasing point. The spectrum shows the peak energy of $33.4 \mathrm{MeV}$ and energy spread of $4.4 \%$ containing $\sim 7 \mathrm{pC}$ of charge.

The transverse slice of the accelerating field is presented in Fig. 2(a). A wakefield with a ring-shaped has been excited by the LG laser pulse, and the transverse position of the maximum accelerating field is the same as the position of the maximum laser field. The lineout of the accelerating field at two transverse positions [labeled by the dashed lines in Fig. 2(a)] is plotted in Fig. 2(b). Figure 2(c) shows the radial slice of the focusing field $E_{y}-B_{x}$ along the $y$ direction, and lineouts of the fields [labeled 

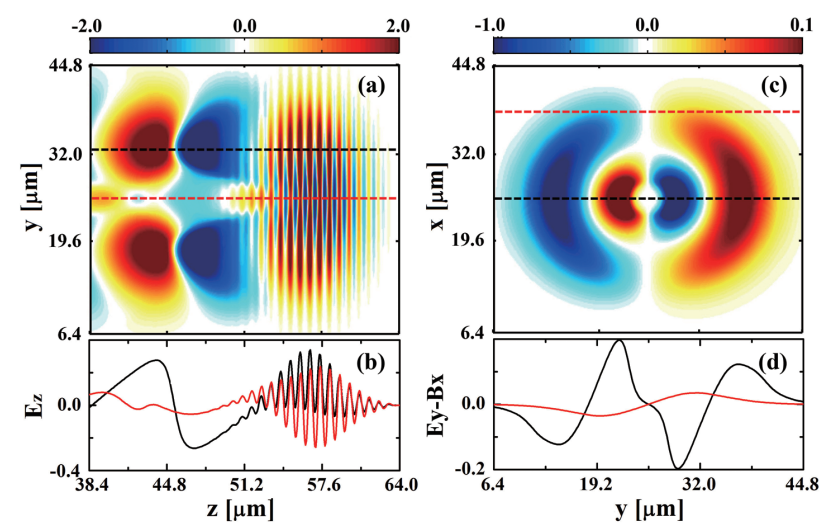

FIG. 2: (color online) Spatial distribution of accelerating field $E_{z}$ in the plane of $x=25.6 \mu \mathrm{m}$ (a) and spatial distribution of focusing field $E_{y}-B_{x}$ at the plane of $z=51.2 \mu \mathrm{m}$ (c). The time is $t=126 \mathrm{fs}$ and the normalized field unit is $804 \mathrm{GV} / \mathrm{m}$. The red and black lines shown in (b) and (d) represent the electric fields corresponding to the positions of the dashed lines as shown in (a) and (c), respectively.

by the dashed lines in Fig. 2(c)] are shown in Fig. 2(d). One can see four crescents-shaped focusing field structures. These structures can confine the electrons within a ring-shaped structure and affect the beam shape evolution. The electron motion results from a balance between the radial force from these focusing fields and the angular momentum from the residual ionization momentum. We also find that there is a relatively weak accelerating field about $44 \mathrm{GV} / \mathrm{m}$ in the center axis and that non-zero focusing fields are present near the axis. These fields make electron acceleration along the axis possible. Our simulations show that, with some parameters, electrons from self-injection can be accelerated along the axis forming a central beam and the ionization-injected electrons are accelerated in the wake forming a ring beam nearby. These two beams have well-seperated peak energies due to the different intensities of the longitudinal fields.

To describe the dynamics of the hollow electron beam, the evolution of the ring size and beam thickness of the accelerated electrons are studied. The radius of the ring is defined by the transverse centroid value of the electrons inside the ring as $r_{c}=\sum_{1}^{N} r_{i} / N$, where $r_{i}$ is the radial position of each electron, $N$ is the total electron number. The temporal evolution of the ring radius $r_{c}$ is shown in Fig. 3 by the red circles. The initial ring radius is $r_{c}=7.235 \mu \mathrm{m}$, which is close to the transverse position of the peak wakefield, i.e., $r_{m}=w_{0} / \sqrt{2}=7.21 \mu \mathrm{m}$. During the acceleration, the ring radius performs oscillations. Related to betatron oscillation, the oscillation period is longer at higher electron energies. The betatron frequency of spherical blowout can be expressed $\omega_{\beta}=\omega_{p} / \sqrt{2 \gamma}$, where $\omega_{p}$ is the plasma frequency and $\gamma$ is the Lorentz factor of the electron beam. Therefore, the betatron wavelength should be $\lambda_{\beta}=\lambda_{p} \sqrt{2 \gamma}=17.7 \sqrt{\gamma}[\mu \mathrm{m}]$. In our simulation, the second oscillation period is about $300 \mathrm{fs}$,

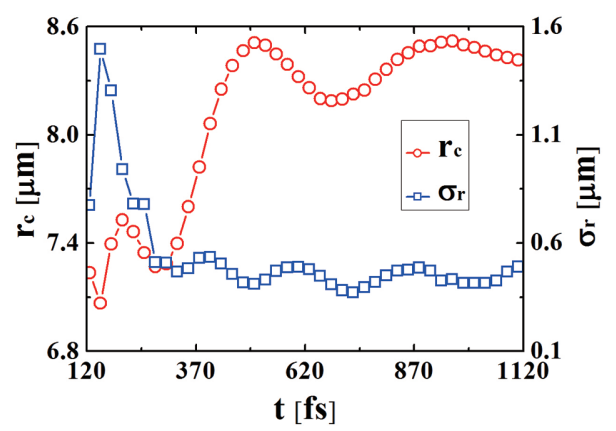

FIG. 3: (color online) The evolution of electron ring radius (red circles) and thickness (blue squares).

which close to the theory estimate $309 \mathrm{fs}$. One can also see that the ring radius here rapidly goes up from $t=277 \mathrm{fs}$ to $t=504 \mathrm{fs}$. This is due to the driver pulse expansion in the radial direction during this period. After this initial time, the laser transitions to a more stable propagation.

We define the beam thickness as the root-meansquare (RMS) of the electron radial distribution $\sigma_{r}=$ $\sqrt{\sum_{1}^{N}\left(r_{i}-r_{c}\right)^{2} / N}$, which is also shown by the blue squares in Fig. 3. During the initial injection time (126fs $<t<176 \mathrm{fs}$ ), the beam thickness is larger because ionization injection is on-going and the electrons have not slipped to the back of the wake yet. Later the thickness of the beam shows periodic oscillation from the betatron oscillations in the wake. Meanwhile, the oscillation period is close to half of the ring centroid value oscillation.

We also find that due to the asymmetry originating from the driver pulse linear polarization, the ring beam distribution shows non-axisymmetric characteristics. To describe the dynamics of the hollow electron beam, we divided the electron ring azimuthal section$\mathrm{s}$, each occupying a limited azimuthal angle region of $\theta_{i}-5^{\circ}<\overline{\theta_{i}}<\theta_{i}+5^{\circ}$. The temporal evolution of the hollow electron beam angular distribution is shown in Fig. 4. The red point position shows the local (within an angle bin) beam center, its size represents the local electron charge, and the blue error bars show the local beam thickness. At the beginning of ionization injection [shown in Fig. 4(a)], the ionized electrons show an annulus ringshaped with ring radius of $r_{c}=7.24 \mu \mathrm{m}$ and the charge density is relatively uniform. However, as a consequence of laser field ionization, the injected electrons always carry initial residual momenta from the laser vector, which is usually one of the main emittance sources of wakefield accelerated electrons from ionization injection $[8,9,24]$. Therefore, the electrons tend to move along the laser polarization direction later as shown in Fig. 4(c). Once the electrons are completely trapped by the wakefield, the electron dynamics will be determined by both the initial residual ionization momentum and the wakefield focusing force. The electron ring radius gradually increases and the shape of the ring gradually changes. As one can 

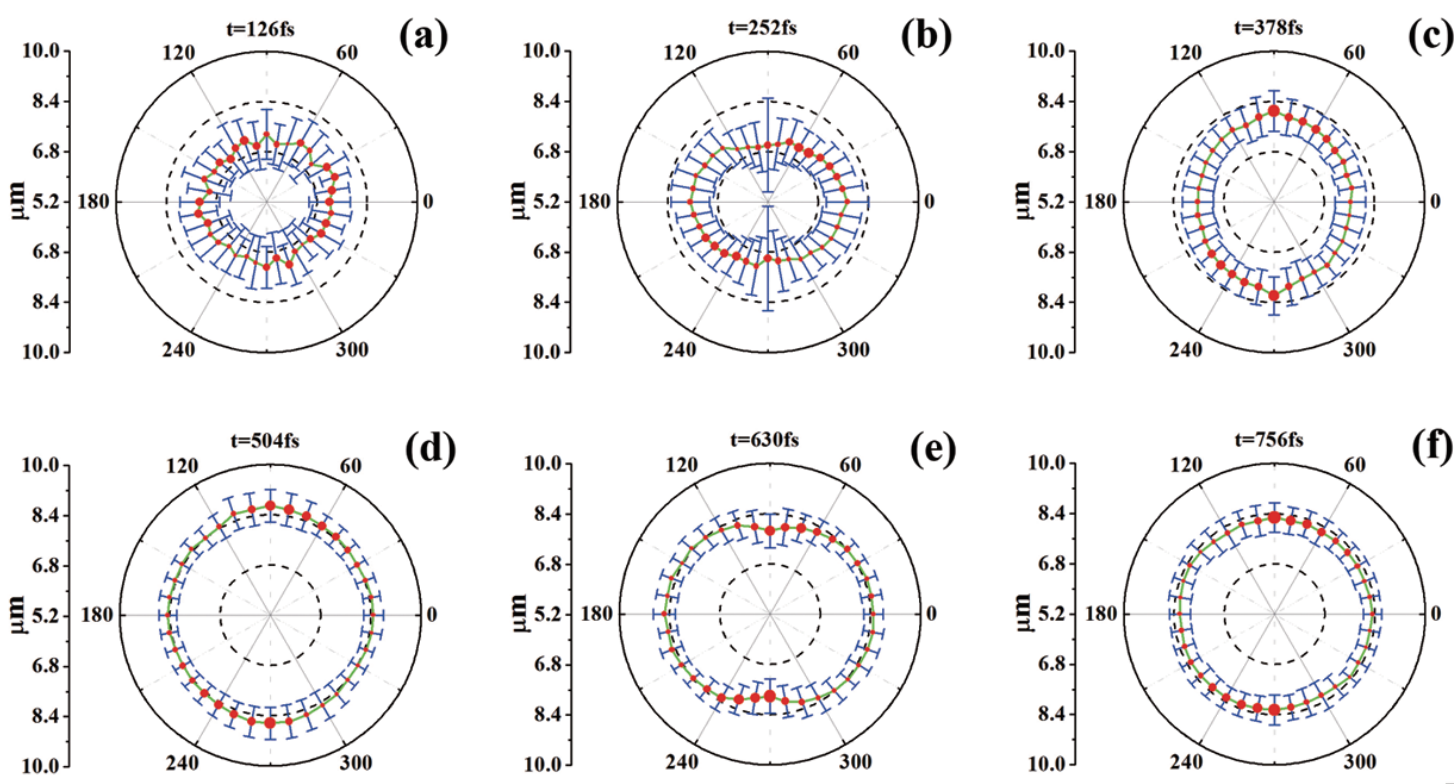

(f)
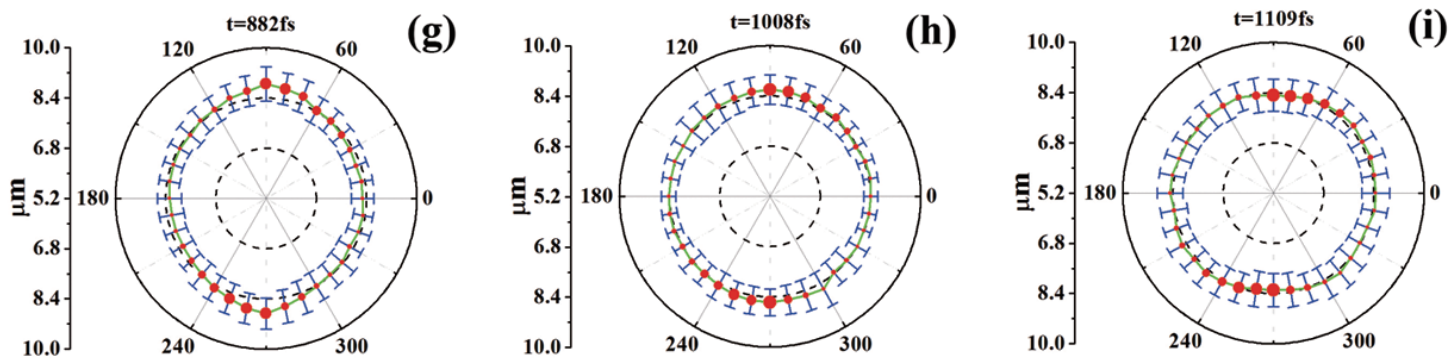

(i)

FIG. 4: (color online) Spatial distributions of the electron ring structure at different acceleration instant. The radius of the beam is represented by the green line, the thickness is represented by the blue error bars and the electron charge is represented by the size of the red points.

see, although the local thickness and the ring radius are approximately uniform at the end of the simulation, the charge distribution remains asymmetric. Electrons move along the laser polarization and pile up at the end, which leads to the asymmetric electron charge distribution as shown in Fig. 4(i).

To describe the electron ring evolution, we examine the beam ellipticity by considering the ratio between the two perpendicular beam radii, $\rho=r_{0^{\circ}} / r_{90^{\circ}} \approx r_{180^{\circ}} / r_{270^{\circ}}$. As shown in Fig. 5, this ratio oscillates near $\rho=1$. The hollow electron beam periodically changes its orientation. This indicates that the betatron oscillations along $x$ and $y$ directions are not in phase.

The beam shape evolution is related to the rotation of the electrons along the ring. To see the rotation process, the evolution of electron angular momenta along different azimuthal angles is shown in Fig. 6. Here, we define positive value as right-handed rotation along the hollow electron beam propagation direction, and negative value is left-handed rotation. At $t=126 \mathrm{fs}$ shown in Fig. 6(a), two groups of electrons with helical orbits having opposite handedness are ionized by the LG laser and injected into the wakefield. The electrons primarily have parallel momenta along the laser polarization, as shown in Fig. $7(\mathrm{a})$. Then, as the ionized electrons are trapped by the ring-shaped wakefield, they begin to rotate due to the initial ionization momenta and the wakefield focusing force. Electrons at different positions have different rotation directions. Figures $6(\mathrm{~b})$ and $6(\mathrm{c})$ show that electrons located in the first quadrant and the third quadrant present right-handed rotation, while the ones in the second quadrant and the fourth quadrant present left-handed rotation. In other words, electrons with angles ranging from $\theta=0^{\circ}$ to $\theta=180^{\circ}$ move towards $\theta=90^{\circ}$ and electrons with angles ranging from $\theta=180^{\circ}$ to $\theta=360^{\circ}$ move towards $\theta=270^{\circ}$. This results in the high-charge density regions along the electron ring. When the electrons go into the dephasing phase, the electrons interact with the 


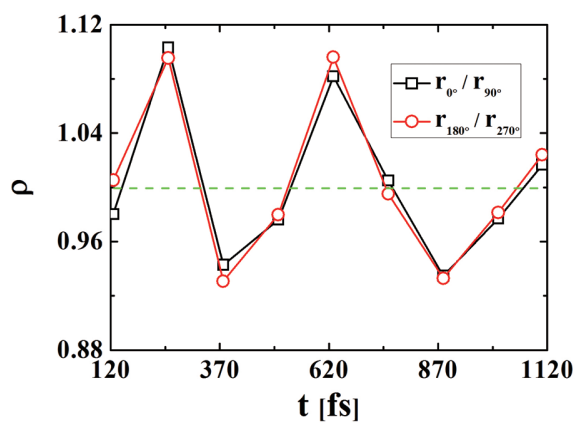

FIG. 5: The ellipticity evolution of the hollow electron beam. Black line represents $\rho=r_{0^{\circ}} / r_{90^{\circ}}$, and red line represents $\rho=r_{180^{\circ}} / r_{270^{\circ}}$. The green dashed line represents $\rho=1$ corresponding to a uniformly distributed circular hollow electron beam.
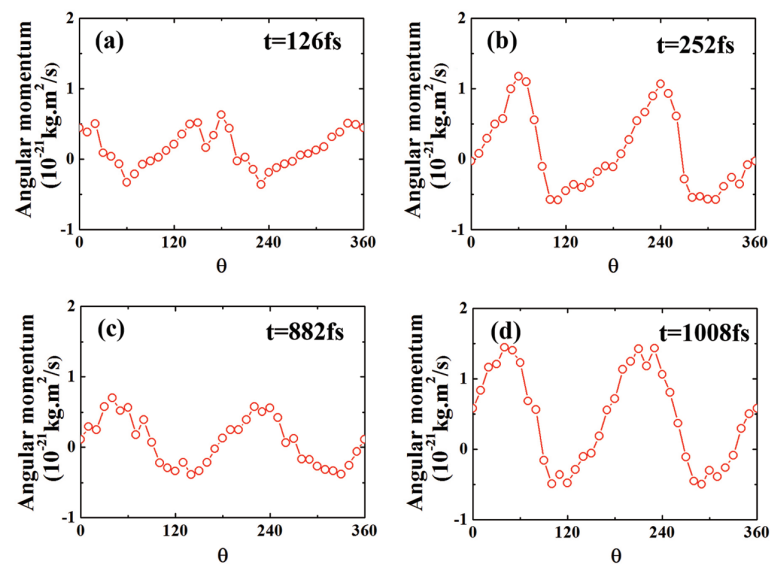

FIG. 6: (color online) Azimuthal angular momentum distribution of electrons at different times.

laser field and obtain transverse momenta again from the LG pulse [as shown in Fig. 6(d)]. The angular momentum with right-handed rotation increases.

To show the effects of wakefields and initial angular momenta from the drive laser pulse on the beam rotation, we plot the electron initial momenta distribution and their total angular momenta evolution in Fig. 7. From Fig. 7(a) one can see that, a hollow electron beam is initially ionized by the LG pulse. The electrons inside the ring primarily have parallel momenta along the laser polarization direction. Figure $7(\mathrm{~b})$ shows the evolution of the total angular momentum of the hollow electron beam. The $\mathrm{LG}_{0}^{1}$ mode laser pulse carries total angular momentum[21] of:

$$
L_{a m}=\left(a_{0}^{2} \times 1.37 \times 10^{18} \mathrm{~W} / \mathrm{cm}^{2}\right) w_{0}^{2} \tau T / 2
$$

where $T$ is the laser period. In our simulation, the total laser angular momentum is $L_{a m}=1.3 \times 10^{-16} \mathrm{~kg} . \mathrm{m}^{2} / \mathrm{s}$. The Nitrogen gas is ionized by LG laser at $t=126 \mathrm{fs}$, and the total angular momentum of the hollow electron beam
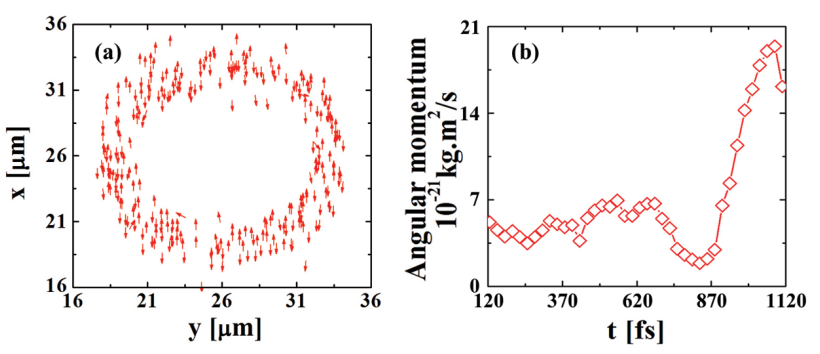

FIG. 7: (color online) (a) Momentum vector distribution of the injected electrons at the initially ionization time of $t=126 \mathrm{fs}$. (b) Total angular momentum of electron ring plotted as a function of the time evolution.

$\left(x p_{y}-y p_{x}\right)$ is about $5 \times 10^{-21} \mathrm{~kg} . \mathrm{m}^{2} / \mathrm{s}$. As one can see that the transfer efficiency from the laser pulse to the accelerated hollow electron beam is very low. However, it is not negligible for the electrons' dynamics as we have discussed before. We find that, although the beam evolves strongly during acceleration, the total angular momentum of the hollow electron beam is almost constant until the electrons reach the dephasing point, at $t=872 \mathrm{fs}$, and interact with the drive laser pulse. Then the total angular momenta of the hollow electron beam gradually increases.

\section{RING RADIUS DEPENDENCY ON LASER AND PLASMA PARAMETERS}

In this section, we study the effects of laser and plasma parameters on the hollow electron beam ring radius and the trapped electron charge. To generate highquality hollow electron beam, a wake with a well-defined ring-shaped structure should be generated. In previous work, an analytical theory is used to predict the condition for ring-shaped wakefield generation in the blowout regime[18]. Considering the usual blowout matching condition $k_{p} r_{b}=2 \sqrt{a_{0}}$, the bubble inner radius should satisfy $r_{b}<r_{m}$ to obtain a well-defined ring-shaped wakefield structure, where $r_{b}$ is the bubble radius and $k_{p}$ is plasma wake number. Thus, an approximate condition to generate an electron ring is $k_{p} w_{0}>2 \sqrt{2 a_{0}}$. In our simulation, the parameters satisfied $k_{p} w_{0} \approx 5>2 \sqrt{2 a_{0}}=4$. Figure 8 (a) shows the laser spot size effects on the electron ring radius $r_{c}$ and the trapped electron charge $Q$ at $t=454 \mathrm{fs}$. One can see that the ring structure cannot be produced until the laser spot size is larger than $6.4 \mu \mathrm{m}$, which is close but smaller than the prediction above $(7.96 \mu \mathrm{m})$. When the laser spot size is smaller than this value, we find the ring-shaped wake structure is not well-defined and the on-axis wakefield is nonzero. Although the initial ionized electrons are also annularly distributed, the transversely oscillating electrons are very easily injected into the axial accelerating field and finally a single electron beam on axis is produced. With the increase of the laser focal spot, the ring-shaped wake structure appears 

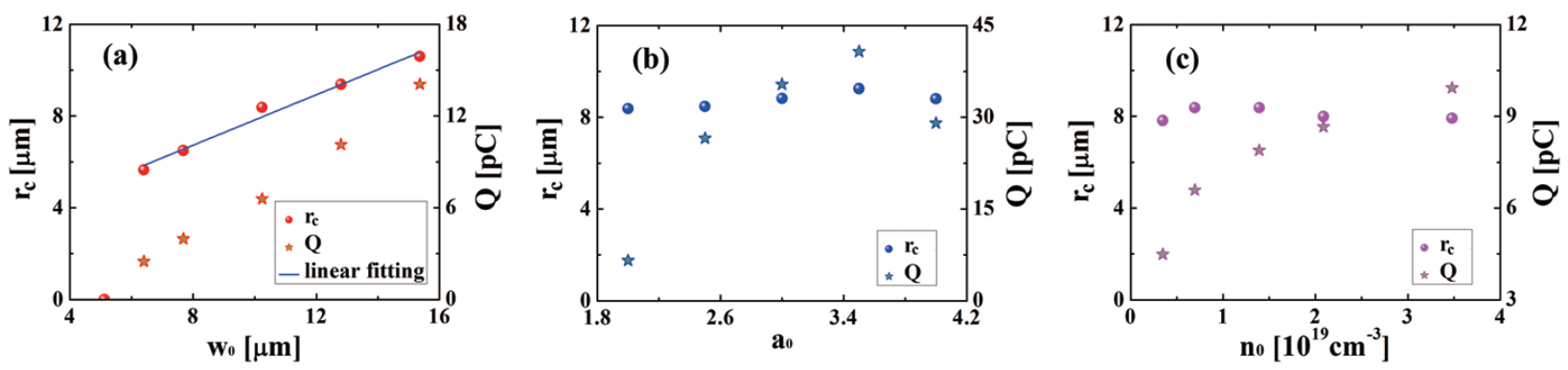

FIG. 8: (color online) 3D-PIC simulation results of the electron ring radius and the trapped electron charge at $t=454 \mathrm{fs}$ for varying laser spot size (a), laser intensity (b), and initial hydrogen plasma density (c). The blue line in (a) is the linear fitting curve.

and the electron ring radius and the trapped electron charge increase gradually as shown in Fig. 8(a). A linear fitting curve is also shown (blue line) in Fig. 8(a). The fitting curve can be expressed as $r_{c}=0.55 w_{0}+2.33$. This linear relationship makes it possible to control the size and the charge of the beam ring by adjusting the laser spot size. Unlike the laser spot size, we found the ring size is only weakly dependent on the laser intensity, as shown in Fig. 8(b). However, the laser intensity should be larger than the threshold for ionization injection. And as the laser intensity enlarges, the trapped electron charge gradually increases at first. Then, it decreases originating from large number of the transversely oscillating electrons injected into the axis accelerating field. A hollow electron beam and an axis electron beam are produced simultaneously. Similarly, Fig. 8(c) shows that the ring radius is also weakly dependent on the plasma density. Meanwhile, the trapped electron charge also increase with initial hydrogen plasma density. Both of these dependencies are reasonable since the location of the maximum laser intensity along the transverse direction determines the maximum radial position of the wake structure. The ring radius strongly depends on the location of the wakefield maximum.

\section{SUMMARY AND DISCUSSION}

In summary, we have studied the acceleration and evolution of a hollow relativistic electron beam in a laser wakefield driven by a LG laser pulse. The LG laser pulse, which has transverse ring intensity-distribution, can produce a ring-shaped wakefield. Part of the angular momentum of the LG laser is transferred to the ionizationinjected electrons. The angular momentum will result in rotation of the electrons in the wakefield. However, electrons from different positions of the hollow ring experience different rotation orientations because of the laser polarization and wakefield focusing forces. Due to the small initial transverse momenta, most of the ionization injected electrons cannot make multi circle helical motions in the wakefield. Electrons in the ring move along the direction of the laser polarization piling up at the end and form an electron ring with non-uniform density distribution. Scaled simulations show that the ring size and the trapped electron charge of the hollow electron beam can be well controlled by tuning the laser spot size; however, both the laser intensity and plasma density have a weak effect on the ring size.

In some other simulations, we found by using external injected electrons with initial larger transverse momenta the electrons may do multi circle helical motions around the laser axis once the balance of the transverse momenta and the transverse wake focusing fields is satisfied. In a simulation, with laser parameters of $a_{0}=1.7$, $w_{0}=4 \mu \mathrm{m}, \tau=10 \mathrm{fs}$ and background plasma with density $8.7 \times 10^{18} \mathrm{~cm}^{-3}$, an initially injected electron beam with momenta of $p_{z}=15, p_{y}=4$, and $p_{x}=0$ have been observed to make helical motions with screwy radius about $3 \mu \mathrm{m}$. Such electron motion makes a circularly polarized hollow x-ray source possible, which could be a simpler alternate radiation scheme to other ones for similar sources.[28]

Besides radiation source, such a hollow electron beam structure may also have applications for accelerating and collimating positively charged particles, and so on. We expect that the generation of tailored beam shapes in LWFAs by using laser drivers with unique modes will open a wide range of potential applications.

\section{Acknowledgments}

This work was supported by the National Basic Research Program of China (Grant No. 2013CBA01504), the National Science Foundation of China (Grant Nos. 11374209, 11374210, 11475260, 11375265), a Leverhulme Trust Research Project Grant, the U.S. Department of Energy under Contracts No. DE-AC02-05CH11231, and by the DOE SCIDAC program COMPASS. M.C. appreciates the support from national 1000 Youth Talent Project of China. Simulations were performed on the $\Pi$ supercomputer at Shanghai Jiao Tong University and Tianhe II supercomputer at Guangzhou. 
[1] T. Tajima, and J. M. Dawson, Phys. Rev. Lett. 43, 267 (1979).

[2] E. Esarey, C. B. Schroeder, and W. P. Leemans, Rev. Mod. Phys. 81, 1229 (2009).

[3] D. Strickland and G. A. Mourou, Opt. Commum. 56, 219 (1985).

[4] H. T. Kim, K. H. Pae, H. J. Cha, I. J. Kim, T. J. Yu, J. H. Sung, S. K. Lee, T. M. Jeong, and J. Lee, Phys. Rev. Lett. 111, 165002 (2013).

[5] W. P. Leemans, A. J. Gonsalves, H. S. Mao, K. Nakamura, C. Benedetti, C. B. Schroeder, Cs. Tóth, J. Daniels, D. E. Mittelberger, S. S. Bulanov, J. L. Vay, C. G. R. Geddes, and E. Esarey, Phys. Rev. Lett. 113, 245002 (2014).

[6] J. Faure, C. Rechatin, A. Norlin, A. Lifschitz, Y. Glinec and V. Malka, Nature 444, 737-739 (2006).

[7] C. Rechatin, J. Faure, A. Ben-Ismail, J. Lim, R. Fitour, A. Specka, H. Videau, A. Tafzi, F. Burgy, and V. Malka, Phys. Rev. Lett. 102, 164801 (2009).

[8] L. L. Yu, E. Esarey, C. B. Schroeder, J. L. Vay, C. Benedetti, C. G. R. Geddes, M. Chen, and W. P. Leemans, Phys. Rev. Lett. 112, 025001 (2014).

[9] C. B. Schroeder, J. L. Vay, E. Esarey, S. S. Bulanov, C. Benedetti, L. L. Yu, M. Chen, C. G. R. Geddes, and W. P. Leemans, Phys. Rev. ST Accel. Beams 17, 101301 (2014).

[10] M. Zeng, M. Chen, L. L. Yu, W. B. Mori, Z. M. Sheng, B. Hidding, D. A. Jaroszynski, and J. Zhang, Phys. Rev. Lett. 114, 084801 (2015).

[11] M. Mirzaie, S. Li, M. Zeng, N. A. M. Hafz, M. Chen, G. Y. Li, Q. J. Zhu, H. Liao, T. Sokollik, F. Liu, Y. Y. Ma, L.M. Chen, Z. M. Sheng, and J. Zhang, Sci. Rep. 5, 14659 (2015)

[12] C. Brabetz, S. Busold, T. Cowan, O. Deppert, D. Jahn, O. Kester, M. Roth, D. Schumacher, and V. Bagnoud, Phys. Plasmas 22, 013105 (2015);N. Jain, T. M. Antonsen, and J. P. Palastro, Phys. Rev. Lett. 115, 195001 (2015).

[13] G. Stancari, A. Valishev, G. Annala, G. Kuznetsov, V. Shiltsev, D. A. Still, and L. G. Vorobiev, Phys. Rev. Lett. 107, 084802 (2011).

[14] J. J. Ramirez, D. E. Hasti, J. P. Corley, J. W. Poukey, K. R. Prestwich, P. W. Spence, I. D. Smith, L. G. Schlitt, H. N. Nishimoto, and K. E. Nielsen, "The four stage helia experiment," in Proceedings of the 5th IEEE Pulsed Power Conference, Arlington, Virginia,10-12 June (1985).

[15] A. A. Kim, B. M. Kovalchuk, A. N. Bastrikov, V. G. Durakov, S. N. Volkov, and V. A. Sinehryukhov, "100 ns current rise time LTD stage," in Proceedings of the 2001 IEEE Pulsed Power Plasma Sciences Conference, available online from IEEE Explore Document No. 0-78037120-8102.

[16] E. Cormier-Michel, E. Esarey, C. G. R. Geddes, C. B. Schroeder, K. Paul,P. J. Mullowney, J. R. Cary, and W. P. Leemans, Phys. Rev. ST Accel. Beams 14, 031303 (2011).

[17] L. L. Yu, C. B. Schroeder, F. Y. Li, C. Benedetti, M. Chen, S. M. Weng, Z. M. Sheng, and E. Esarey, Phys. Plasmas 21, 120702 (2014).

[18] J. Vieira, and J. T. Mendonça, Phys. Rev. Lett. 112, 215001 (2014)

[19] Y. Shi, B. F. Shen, L. G. Zhang, X. M. Zhang, W. P. Wang, and Z. Z. Xu, Phys. Rev. Lett. 112, 235001 (2014).

[20] X. M. Zhang, B. F. Shen, Y. Shi, X. F. Wang, L. G. Zhang, W. P. Wang, J. C. Xu, L. Q. Yi, and Z. Z. Xu, Phys. Rev. Lett. 114, 173901 (2015).

[21] W. P. Wang, B. F. Shen, X. M. Zhang, L. G. Zhang, Y. Shi and Z. Z. Xu, Sci. Rep. 5, 8274 (2015).

[22] J. T. Mendonça and J. Vieira, Phys. Plasmas 21, 033107 (2014).

[23] M. Chen, Z. M. Sheng, Y. Y. Ma, and J. Zhang, J. Appl. Phys. 99, 056109 (2006).

[24] M. Chen, E. Esarey, C. B. Schroeder, C. G. R. Geddes, and W. P. Leemans, Phys. Plasmas 19, 033101 (2012).

[25] C. Thaury, E. Guillaume, S. Corde, R. Lehe, M. Le Bouteiller, K. Ta Phuoc, X. Davoine, J. M. Rax, A. Rousse, and V. Malka, Phys. Rev. Lett. 111, 135002 (2013).

[26] M. Chen, E. Cormier-Michel, C. G. R. Geddes, D. L. Bruhwiler, L. L. Yu, E. Esarey, C. B. Schroeder, W. P. Leemans, J. Comput. Phys. 236, 220-228 (2013).

[27] A. Pak, K. A. Marsh, S. F. Martins, W. Lu, W. B. Mori, and C. Joshi, Phys. Rev. Lett. 104, 025003 (2010).

[28] M. Chen, J. Luo, F.-Y. Li, F. Liu, Z.-M. Sheng, and J. Zhang, Light Sci. Appl. 5, e16015 (2016). 